\title{
Sum rules among Higgs gauge couplings in the electroweak chiral Lagrangian.
}

\section{Ryo Nagai}

Nagoya University

E-mail: nagaieth.phys.nagova-u.ac.ip

In the framework of the electroweak chiral Lagrangian, we (re)derive sum rules for the Higgs gauge couplings by requiring following two conditions. The first is the cancellation of bad high energy behavior in the longitudinal electroweak gauge boson scattering amplitudes. The second is the finiteness of the oblique correction parameter, $S, T$ and $U$. Using the sum rules, we evaluate upper mass bounds on the neutral, singly -charged and doubly-charged scalar bosons as a function of the deviation of the SM-like Higgs gauge coupling.

KMI International Symposium 2013 on "Quest for the Origin of Particles and the Universe" 11-13 December, 2013

Nagoya University, Japan 


\section{Introduction}

The last year discovery of a Higgs boson at $125 \mathrm{GeV}$ at the Large Hadron Collider (LHC) experiments completed the set of all particles predicted in the Standard Model (SM). We now have a key particle to solve the mystery of the origin of particle masses (electroweak symmetry breaking). One of the key roles of the SM Higgs boson is to unitarize the high energy longitudinal weak gauge bosons' scattering amplitudes. The Higgs boson also makes the SM renormalizable, i.e., it cancels non-renormalizable ultraviolet divergences appearing at the loop level. In order to make the SM unitary and renormalizable, the Higgs coupling strengths need to be precisely adjusted. If the measured value of Higgs coupling strengths turn out to deviate from the SM values, in order to make the theory unitary and consistent with the electroweak precision tests, new particles other than the SMlike Higgs boson need to exist. Can we make definite predictions for the properties of this required new particle (new "Higgs" boson) in this case? In our study[龱, we try to answer this question from the viewpoint of the unitarity and the electroweak precision tests as model independent as possible.

\section{Framework}

We use the electroweak chiral Lagrangian technique to describe the arbitrary interactions among weak gauge bosons and "Higgs" bosons in an $S U(2) \times U(1)$ gauge inavriant manner. The interaction Lagrangian terms relevant for following discussions are given by

$$
\begin{aligned}
\mathscr{L} & =-v \sum_{n=1}^{N_{0}} \kappa_{W}^{\phi_{n}^{0}} \phi_{n}^{0} \operatorname{tr}\left[U^{\dagger} D_{\mu} U \tau_{+}\right] \operatorname{tr}\left[U^{\dagger} D^{\mu} U \tau_{-}\right] \\
& -\frac{v}{4} \sum_{n=1}^{N_{0}} \kappa_{Z}^{\phi_{n}^{0}} \phi_{n}^{0} \operatorname{tr}\left[U^{\dagger} D_{\mu} U \tau_{3}\right] \operatorname{tr}\left[U^{\dagger} D^{\mu} U \tau_{3}\right] \\
& -\frac{v}{\sqrt{2}} \sum_{n=1}^{N_{+}} \kappa_{W Z}^{\phi_{n}^{-}} \phi_{n}^{-} \operatorname{tr}\left[U^{\dagger} D_{\mu} U \tau_{-}\right] \operatorname{tr}\left[U^{\dagger} D^{\mu} U \tau_{3}\right]+\text { h.c. } \\
& -\frac{v}{2} \sum_{n=1}^{N_{+}} \kappa_{W W}^{\phi_{n}^{--}} \phi_{n}^{--} \operatorname{tr}\left[U^{\dagger} D_{\mu} U \tau_{-}\right] \operatorname{tr}\left[U^{\dagger} D^{\mu} U \tau_{-}\right]+\text {h.c. }+\cdots,
\end{aligned}
$$

with $U$ being the nonlinear sigma model field $U=\exp \left(i w^{a} \tau^{a} / v\right)$ of the electroweak symmetry breaking $[S U(2) \times U(1)] / U(1)$. The Nambu-Goldstone boson fields, the Pauli matrices, and the vacuum expectation value are denoted by $w^{a}, \tau_{a}(a=1,2,3)$, and $v \sim 246 \mathrm{GeV}$, respectively. The SM-like Higgs boson $h \equiv \phi_{1}^{0}$ is introduced as a matter particle in the chiral Lagrangian, so are other neutral and charged "Higgs" bosons $\phi_{n}^{Q}$. These coupling strengths are denoted by coefficients $\kappa$.

\section{Unitarity sum rules}

The cancellation of the unitarity violating high energy scattering amplitudes requires a set of conditions among these Higgs couplings ("unitarity sum rules"), which was first derived in Ref.[వ] (see also [3]). In our study, we rederive the sum rules[[2] in our gauge invariant Lagrangian[eq([2])] using the equivalance theorem between the Nambu-Goldstone boson amplitudes and the longitudinal gauge boson amplitudes. This is in contrast to the original derivation of Ref.[[]], in which the 
Higgs couplings are introduced as Feynnman rules of Higgs vertices without introducing gauge invariant Lagrangians. Requiring the unitarity in $W_{L}^{+} W_{L}^{-} \rightarrow W_{L}^{+} W_{L}^{-}\left(Z_{L} Z_{L}\right)$, for examples, we obtain sum rules among $\kappa$ :

$$
\begin{aligned}
1-\left(\kappa_{W}^{\phi_{1}^{0}}\right)^{2} & =\sum_{n=2}^{N_{0}}\left(\kappa_{W}^{\phi_{n}^{0}}\right)^{2}-\sum_{n=1}^{N_{++}}\left|\kappa_{W W}^{\phi_{n}^{--}}\right|^{2} \\
1-\kappa_{W}^{\phi_{1}^{0}} \kappa_{Z}^{\phi_{1}^{0}} & =\sum_{n=2}^{N_{0}} \kappa_{W}^{\phi_{n}^{0}} \kappa_{Z}^{\phi_{n}^{0}}-\sum_{n=1}^{N_{+}}\left|\kappa_{W Z}^{\phi_{n}^{-}}\right|^{2}
\end{aligned}
$$

These sum rules tell us qualitative information about "Higgs" bosons: If the SM-like Higgs boson's the coupling strength with weak gauge bosons is smaller (larger) than the SM prediction, there should be other neutral (charged) Higgs bosons which interact with weak gauge bosons.

\section{Finiteness conditions for the electroweak oblique corrections}

Thanks to the gauge invariance of our framework[eq([2])], we are able to evaluate one-loop effects to the electroweak oblique corrections (Peskin-Takeuchi parameters $S, T$ and $U$ ). For an example, $T$ parameter is evaluated at one loop as

$$
\begin{aligned}
T & =\frac{\sqrt{2} G_{F}}{16 \pi^{2} \alpha} \sum_{n=1}\left[\left\{\left(\kappa_{Z}^{\phi_{n}^{0}}+2 \kappa_{W}^{\phi_{n}^{0}}\right)\left(\kappa_{Z}^{\phi_{n}^{0}}-\kappa_{W}^{\phi_{n}^{0}}\right)\right\} \Lambda^{2}\right. \\
& \left.+3 \kappa_{Z}^{\phi_{n}^{0}}\left(\kappa_{Z}^{\phi_{n}^{0}}-\kappa_{W}^{\phi_{n}^{0}}\right) m_{Z}^{2} \ln \Lambda^{2}+6 \kappa_{W}^{\phi_{n}^{0}}\left(\kappa_{Z}^{\phi_{n}^{0}}-\kappa_{W}^{\phi_{n}^{0}}\right) m_{W}^{2} \ln \Lambda^{2}\right]+ \text { finite }
\end{aligned}
$$

with $\Lambda$ being the ultraviolet cutoff. We have assumed the the unitarity sum rules in the evaluation of (4. I). In order to ensure the finiteness of $T$ parameter without introducing extra counter terms, $\kappa$ need to satisfy a condition,

$$
\kappa_{W}^{\phi_{n}^{0}}=\kappa_{Z}^{\phi_{n}^{0}}
$$

Similar conditions can also be obtained from the finiteness of $S$ and $U$ parameters.

\section{Constraining non-SM "Higgs" masses}

Note that the sum rules (B.]), (B.2) and the finiteness relation (4.2) are independent of the Higgs bosons' masses. Can't we constrain masses of extra Higgs bosons which are required if the $125 \mathrm{GeV}$ Higgs boson couplings turn out to deviate from the those of the SM Higgs boson? Yes, we can constrain the non-SM "Higgs" masses by using the perturbative unitarity bound[田] and the electroweak precision measurements of $S, T$ and $U$ [ []] under such circummstances. From these requirements, we can evaluate upper bound of extra Higgs boson masses as a function of the deviation of the SM-like Higgs gauge coupling $\Delta \kappa_{V} \equiv \kappa_{W(Z)}^{\phi_{1}^{0}}-1$. Here, for simplicity, we assume that there are only two neutral Higgs bosons [Figure $\mathrm{U}(\mathrm{left})]$. the horizontal axis is the mass of unknown extra Higgs boson, and the vartical axis is the deviation of the SM-like Higgs boson coupling strength with gauge bosons from that of value of the SM prediction. This result show that if the SM-like Higgs boson coupling strength with gauge bosons is 5\% smaller than that of the 

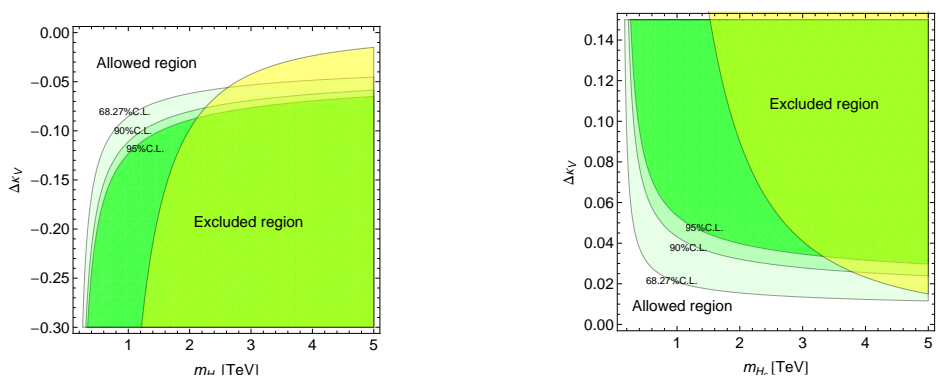

Figure 1: Constraint to the mass of extra neutral Higgs boson (left) and charged Higgs boson (right). Yellow region is excluded region by perturbative unitarity bound[四]. Green region is excluded region by constraint to $S, T$ and $U$ parameters[[]].

SM prediction, Extra higgs boson whose mass is lighter than $3 \mathrm{TeV}$ is needed. For the case that there are one neutral Higgs boson (the SM-like Higgs boson) and mass degenerated charged Higgs bosons, we can also evaluated upper mass bound of charged Higgs bosons in the similar manner [Figure प(right)].

\section{Summary}

In our study, we have (re)derived a set of conditions among coupling strength between "Higgs" bosons and weak gauge bosons to guarantee the unitarity of the scattering amplitudes and ensure the finiteness of elecrtoweak oblique corrections without extra counter terms. These conditions tell us what particle ("Higgs" bosons) are needed if the SM-like Higgs boson's coupling strength with gauge bosons deviate from the SM prediction. Furthermore, if we consider perturbative unitarity bound and consistency with electroweak precision measurements, we can evaluate the upper mass bound of these "Higgs" bosons as a function of the deviation of the SM-like Higgs boson's coupling strength. In these way, Once the deviations of the SM-like Higgs boson coupling strengths are experimentally confirmed in future experiments, these results which we have (re)derived can be used to predict properties of extra particles in the EWSB sector.

\section{Note added}

After the conference, a paper by Grinstein et al. appeared on the arXiv[[]], which also discusses the perturbative unitarity limits in extended Higgs models.

\section{Acknowledgements}

Author is partially supported by the Nagoya University Program for Leading Graduate Schools funded by the Ministry of Education of the Japanese Government under the program number N01. The author is deeply grateful to M. Tanabashi and K.Tsumura , for constructive advice and discussion. 


\section{References}

[1] R. Nagai, M. Tanabashi and K. Tsumura, in preparation.

[2] J. F. Gunion, H. E. Haber and J. Wudka, Phys. Rev. D 43 (1991) 904.

[3] B. Grinstein, C. W. Murphy, D. Pirtskhalava and P. Uttayarat, arXiv:1401.0070 [hep-ph].

[4] B. W. Lee, C. Quigg and H. B. Thacker, Phys. Rev. D 16 (1977) 1519.

[5] M. Baak, M. Goebel, J. Haller et al., Eur. Phys. J. C 72 (2012) 2205 